\title{
Review Article \\ Potential Use of Stem Cells for Kidney Regeneration
}

\author{
Takashi Yokoo, Kei Matsumoto, and Shinya Yokote \\ Project Laboratory for Kidney Regeneration, Institute of DNA Medicine, Department of Internal Medicine, \\ The Jikei University School of Medicine, Tokyo, 105-8461, Japan \\ Correspondence should be addressed to Takashi Yokoo, tyokoo@jikei.ac.jp
}

Received 11 January 2011; Accepted 18 February 2011

Academic Editor: Niels Olsen Saraiva Camara

Copyright () 2011 Takashi Yokoo et al. This is an open access article distributed under the Creative Commons Attribution License, which permits unrestricted use, distribution, and reproduction in any medium, provided the original work is properly cited.

\begin{abstract}
Significant advances have been made in stem cell research over the past decade. A number of nonhematopoietic sources of stem cells (or progenitor cells) have been identified, including endothelial stem cells and neural stem cells. These discoveries have been a major step toward the use of stem cells for potential clinical applications of organ regeneration. Accordingly, kidney regeneration is currently gaining considerable attention to replace kidney dialysis as the ultimate therapeutic strategy for renal failure. However, due to anatomic complications, the kidney is believed to be the hardest organ to regenerate; it is virtually impossible to imagine such a complicated organ being completely rebuilt from pluripotent stem cells by gene or chemical manipulation. Nevertheless, several groups are taking on this big challenge. In this manuscript, current advances in renal stem cell research are reviewed and their usefulness for kidney regeneration discussed. We also reviewed the current knowledge of the emerging field of renal stem cell biology.
\end{abstract}

\section{Introduction}

Recent advances in stem cell research have brought the possibility of using somatic stem cells for organ regeneration one step closer to realization. Newly developed organs can then be used in clinical organ replacement. However, anatomically complicated organs such as the kidney have proven more refractory to stem cell-based regenerative techniques.

The kidney retains the potential to regenerate if the damage is not too severe, and the kidney structure remains intact.

It was previously believed that bone marrow-derived stem cells could differentiate into renal-resident cells and participate in kidney regeneration after renal ischemia/reperfusion injury $[1,2]$; however, recent studies have suggested that the number of bone marrow-derived cells that engraft injured tubules and develop into functional renal tissue is very low and, thus, their overall contribution to renal repair would be minor in the setting of acute kidney injury $[3,4]$. Also, during the progression of chronic renal failure, bone marrow-derived stem cells supplied by intravenous administration located in the kidney and contributed to attenuate renal fibrosis [5]. However, in cases of irreversible damage to the kidney, as can occur with long-term dialysis, the kidney structure is totally lost and, therefore, these cell therapy approach seems not to be applicable. Thus, any application of regenerative medicine in end-stage renal disease will require the de novo development of an entire functional kidney.

In terms of a functional whole kidney, Chan et al. [6] reported the first attempt to develop a functional whole renal unit by developing a transplantable pronephros in Xenopus. Xenopus presumptive ectoderm, which becomes epidermis and neural tissue in normal development, contains pluripotent stem cells and can be differentiated into multilineage tissue cells under particular culture conditions [7]. The researchers designed conditions for the induction of pronephric tubule-like structures from animal caps that involved a combination of activin and retinoic acid for only 3 hours. This pronephros-like tissue was transplanted into bilaterally nephrectomized tadpoles to test for its functional integrity as a pronephros. Bilateral pronephrectomy induces severe edema in tadpoles owing to their inability to excrete internal water, and tadpoles die within 9 days; transplantation of the pronephros-like unit at least partially corrected the edema, and tadpoles survived for up to 1 month. To our knowledge, this study is the only one to 
develop a transplantable functional whole kidney unit in vitro, although the pronephros structure formed was too primitive for any clinical application in humans.

Since then, many attempts have been made worldwide to regenerate whole kidneys applicable for mammals from stem cells. The present article reviews the challenges and recent advances in renal stem cell research and discusses its potential for clinical application for kidney regeneration in humans.

\section{Embryonic Kidney as a Source of Organ Regeneration}

Woolf et al. [8] reported that the metanephros may continue to grow if it is transplanted into the renal cortex of host mice. The developed transplant contains vascularized glomeruli and mature proximal tubules and may have the capacity for glomerular filtration. Collecting duct-like structures appear to extend from the transplant toward the papilla of the host. Although there is no direct evidence that these collecting duct-like structures connect with the host's collecting system or that the transplant functions in a manner similar to that of a native kidney, the results provide a rationale for the existence of renal stem cells in the metanephros from early embryos, which may be a potential source of transplantable regenerated kidney. Potential problems with this system include questions as to the suitability of the renal capsule of dialysis patients as a transplant site, given the significant disruptions to this area, including the vasculature, and the fact that space limitations beneath the renal capsule may hinder the growth of transplants. These concerns may be overcome by the system established by Rogers et al. [9]. These researchers also used the metanephros as a source of transplantable artificial kidney but transplanted the graft into a host omentum, which is not confined by a tight organ capsule or disturbed by dialysis. Metanephroi from rat, mouse, and pig were implanted into the omenta of the rat or mouse, and the success of transplanting across xenogeneic barriers and the extent of differentiation into a functional nephron were evaluated. This experiment was based on previous studies showing minimal immunogenicity in tissues harvested at earlier gestational stages, including the metanephros [10]. In cases of allotransplantation (rat metanephros to rat omentum), transplants assumed a kidney-like shape in situ that was approximately one-third the diameter of the native kidney. Histologically speaking, the transplants contained well-differentiated kidney structures. It is important to note that this transplantation technique can be performed without immunosuppression. With xenotransplantation, the pig metanephros grew and differentiated into renal tissue in the rat omentum, showing glomeruli, proximal tubules, and collecting ducts; however, immunosuppressants were required because without these agents the transplants disappeared soon after transplantation. What is interesting is that the graft pig metanephros was slightly larger in volume (diameter and weight) as compared to that of a normal rat kidney. Furthermore, the transplanted tissue produced urine and, surprisingly, after intact ureteroureterostomy with the ureter of the kidney that was removed, anephric rats started to void and showed a prolonged lifespan [11]. This success holds the promise of a new and practical therapeutic strategy for kidney disease in which a functional renal unit can be established by implanting xenometanephros together with immunosuppression.

Recently, Osafune et al. reported an in vitro culture system in which a single Sall1 highly expressing cell from the metanephric mesenchyme forms a 3D kidney structure consisting of glomeruli and renal tubules [12]. More recently, Unbekandt and Davies reported that single-cell suspensions dissociated from metanephros can reaggregate to form a organotypic renal structure if they are treated with an inhibitor of Rho-associated kinase [13]. These systems are useful for examining mechanisms of renal progenitor differentiation and suggest the possibility of developing a whole kidney from a single stem cell.

\section{Kidney Regeneration Using Artificial Scaffolding}

One possible means of creating an optimal niche for pluripotent stem cells to differentiate into a kidney is to provide an artificial scaffold upon which the stem cells can function. This strategy was used recently by Ott et al. [14] to successfully develop a functional artificial rat heart using a cadaveric heart as the artificial scaffold. A whole-heart scaffold with intact $3 \mathrm{D}$ geometry and vasculature was created by coronary perfusion with detergents into the cadaveric heart. This decellularized heart was then repopulated with neonatal cardiac cells or rat aortic endothelial cells and cultured under physiological conditions to simulate organ maturation [14]. The injected neonatal cardiac cells formed a contractile myocardium, which performed the stroke function.

Cadaveric scaffolds have also been used to develop transplantable liver and lung using mature hepatocyte and alveolar epithelial cells, respectively $[15,16]$. After transplantation of the recellularized grafts, they were successfully functioning as a hepatocyte and gas exchanger. Such an approach is promising for regenerating organs that have a simple architecture. However, pluripotent stem cells should be used if this technique is used for kidney regeneration, because the kidney is composed of multiple cell types, such as tubular epithelial cells and glomerular cells. For this purpose, experts should know which stem cells may selectively differentiate into kidney residential cells. One such candidate is embryonic stem (ES) cells.

ES cells are undifferentiated pluripotent stem cells isolated from the inner cell mass of blastocysts [17]. ES cells can differentiate into several cell types of mesodermal, endodermal, and ectodermal lineages, depending on culture conditions. Because human ES cells can differentiate into kidney structures when injected into immunosuppressed mice $[18,19]$, studies have focused on identifying the precise culture conditions that allow the differentiation of ES cells into renal cells in vitro. To this end, Schuldiner et al. [20] showed that human ES cells cultured with 8 growth factors, 
including hepatocyte growth factor (HGF) and activin A, differentiated into cells expressing WT-1 and renin [19]. More recently, mouse ES cells stably transfected with Wnt4 (Wnt4-ES cells) were differentiated into tubular structures expressing AQP-2 in the presence of HGF and activin A [21]. An ex vivo culture system in which ES cells (or ES-derived cells) were cultured in the developing metanephros was also investigated to determine the capacity of ES cells to differentiate into kidney cells integrated into the kidney structure [22]. ROSA26 ES cells were stimulated with developmental signals in the microenvironment of a developing kidney following injection into a metanephros cultured in vitro. ES cell-derived, $\beta$-galactosidase-positive cells were identified in epithelial structures resembling renal tubules with an efficiency approaching 50\% [22]. Based on these results, Kim and Dressler [23] attempted to identify the nephrogenic growth factors needed to induce differentiation of ES cells into renal epithelial cells. When injected into a developing metanephros, ES cells treated with retinoic acid, activin A, and BMP7 contributed to tubular epithelia with near 100\% efficiency [23]. Furthermore, Vigneau et al. [24] showed that ES cells expressing brachyury, a marker of mesoderm specification, became a renal progenitor population in the presence of activin A. After injection into a developing metanephros, these cells might be incorporated into the blastema cells of the nephrogenic zone. In addition, a single injection of the same cells into developing live newborn mouse kidneys saw them stably integrated into proximal tubules with normal morphology and polarization for 7 months without teratoma formation [24]. Taken together, these data highlight ES cells as a potential source of renal stem cells to be differentiated into renal-resident cells.

Based on this series of studies, Ross and coworkers attempted recently to regenerate an entire kidney using a decellularized cadaveric kidney scaffold [25]. After decellularization, murine ES cells were infused via the renal artery and were found to localize to the vasculature and glomeruli, with subsequent migration into the tubules. Immunohistochemical analysis suggested that the infused cells had differentiated into mature kidney cells. Although renal function is yet to be examined, this approach may be useful for the production of an entire kidney.

To address the adverse effects of immunosuppressants, Lanza et al. [26] attempted to develop a self-kidney unit to eliminate the immune response problem and, therefore, the need for immunosuppression. To generate a histocompatible kidney for artificial organ transplantation, Lanza et al. [26] used a nuclear transplantation technique in which dermal fibroblasts isolated from adult cow were transferred into enucleated bovine oocytes and then transferred nonsurgically into progestin-synchronized recipients. After 6-7 weeks, metanephroi were isolated from embryos, digested using collagenase, and expanded until the desired cell number was obtained by culture in vitro. These cells were then seeded onto a specialized polymer tube, followed by implantation into the same cow from which the cells had been cloned. What is striking is that this renal device seeded with cloned metanephric cells appeared to produce a urinelike liquid, whereas those without cells or those seeded with allogeneic cells did not. Histological analysis of the explant revealed a well-developed renal structure composed of organized glomeruli-like, tubule-like, vascular elements that were clearly distinct from one another but continuous within the structure. Therefore, these renal tissues appeared integrally connected in a unidirectional manner to the reservoirs, resulting in the excretion of urine into the collecting system. Although it is not clear how the cultured cells digested from the metanephros gained polarity and self-assembled into glomeruli and tubules, this technique successfully used nuclear transplantation for renal regeneration without the risks and long-term effects of immunosuppression.

\section{De Novo Kidney Regeneration Using Blastocyst Complementation}

It was previously reported that injection of normal ES cells into the blastocysts of recombination-activating gene 2 (RAG-2)-deficient mice, which have no mature B or $\mathrm{T}$ lymphocytes, generated somatic chimeras with ES cellderived mature B and T cells [27]. This blastocyst complementation system was recently applied to reconstructing the entire human kidney. Injection of wild-type ES cells into the blastocysts of Sall1-null mice, which lack kidneys, generated metanephroi composed exclusively of ES cell-derived differentiated cells. This strategy, however, was assessed to be unsuitable for human clinical application, because it is quite difficult to generate interspecific chimeras in livestock. Many groups have sought to generate interspecific chimeras between mouse and rat, achieving success with chimeric preimplantation embryos in vitro but failing with live chimeric animals $[28,29]$. Extraembryonic lineage cells like trophectoderm, derived from xenogenic embryos, may suffer from inhibition of implantation on exposure to the host uterus, suggesting that only cells of preblastocyst origin can contribute to the extraembryonic lineage cells. In this context, the discovery of induced pluripotent stem (iPS) cells allayed this concern.

iPS cells were produced by Takahashi and Yamanaka [30] from cultured somatic cells by retroviral transfer with Oct3/4, Sox2, c-Myc, and Klf4, which are transcription factors associated with pluripotency [31]. Rate-reprogrammed iPS cells were subsequently derived by the reactivation of Fbx15 [31], Oct4 [31], or Nanog [32], all of which carry a drug resistance marker inserted into the respective endogenous locus by homologous recombination or a transgene containing the Nanog promoter. Furthermore, iPS cells were also isolated based on their ES-like morphology, without the use of transgenic donor cells [33], and the therapeutic potential of autologous iPS cells in a mouse model of a hereditary disease has been reported [34]. Recently, human iPS cells were successfully induced from adult skin fibroblasts using the same 4 factors as detailed above [35]. iPS cells are epigenetically and biologically indistinguishable from normal ES cells, and, therefore, it is suggested that they be used to produce interspecific chimeras. Kobayashi and coworkers recently reported successful regeneration of rat pancreas in mouse via an interspecific blastocyst injection 
of iPS cells [36]. They injected rat iPS cells into Pdx-1-/(pancreatogenesis-disabled) mouse blastocysts and found that the newborn chimera of rat and mouse processed almost entirely iPS-derived pancreas. This success proves that when an empty developmental niche for an organ is provided (as with the $\mathrm{Pdx}^{-/-}$mouse and the pancreatic niche), iPS cell-derived cellular progeny can occupy that niche and can compensate developmentally for the missing contents of the niche, forming a complicated organ composed almost entirely of cells derived from donor iPS cells even if the blastocyte complementation involves different species.

Furthermore, instead of tetraploid complementation, Espejel and coworkers generated chimeric mice that experienced progressive liver repopulation with iPS cellderived hepatocytes postnatally using blastocytes deficient in fumarylacetoacetate hydrolase [37]. The entire liver was composed of iPS cell-derived hepatocytes by the time the mice reached adulthood. They replicated the unique proliferative capabilities of normal hepatocytes and were able to regenerate liver after transplantation and two-thirds partial hepatectomy. These successes strongly suggest that blastocyst complementation is one of the most promising strategies for regenerating the kidney. However, these systems are not available for clinical use at this time, because it is not possible to generate the vascular and nervous systems. In addition, the more important ethical issues involved with manipulating blastocysts with iPS cells remain unresolved. Nonetheless, this success highlights the rationale that the eventual clinical application of kidney regeneration must depend on developmental programming.

\section{De Novo Kidney Regeneration Using Growing Xenoembryos}

We attempted to develop an entire kidney using scaffolding from mesenchymal stem cells (MSCs). It has been shown that MSCs have the capacity for site-specific differentiation into various cell types of mesodermal lineage, including chondrocytes, adipocytes, myocytes, cardiomyocytes, bone marrow stromal cells, and thymic stromal cells [38, 39]; this suggests that they may also differentiate into kidney residential cells.

Initially, we tried to reconstruct an organized and functional kidney structure using a developing heterozoic embryo as an "organ factory." During embryogenesis, a single fertilized cell develops into a whole body within 266 (for humans) or 20 days (for rodents). This neonate has every organ positioned correctly, indicating that a single fertilized ovum contains a blueprint from which the body, including the kidney, can be built. Therefore, we sought to "borrow" this programming of a developing embryo by applying the stem cells at the niche of organogenesis.

During development of the metanephros, the metanephric mesenchyme initially forms from the caudal portion of the nephrogenic cord [40] and secretes glial cell linederived neurotrophic factor (GDNF), which induces the nearby Wolffian duct to produce a ureteric bud [41]. The metanephric mesenchyme consequently forms the glomerulus, proximal tubule, loop of Henle, and distal tubule, as well as the interstitium, as a result of reciprocal epithelial-mesenchymal induction between the ureteric bud and metanephric mesenchyme [42]. For this epithelialmesenchymal induction to occur, GDNF must interact with its receptor, c-ret, which is expressed in the Wolffian duct [42]. We hypothesized that GDNF-expressing MSCs may differentiate into kidney structures if positioned at the budding site and stimulated by numerous factors spatially and temporally identical to those found in the developmental milieu.

To investigate this hypothesis, we initially injected human mesenchymal stem cells (hMSCs) into the developing metanephros in vitro, although this was not sufficient to achieve kidney organogenesis [43]. This suggests that hMSCs must be placed before the metanephros begins to develop in a specific, defined embryonic niche to allow their exposure to the repertoire of nephrogenic signals required to generate the organ. This can be best achieved by implanting hMSCs into the nephrogenic site of a developing embryo. Therefore, we established a culture system in combination with a wholeembryo culture system, followed by metanephric organ culture (Figure 1). This "relay culture" allows the development of the metanephros from structures present before budding until the occurrence of complete organogenesis ex utero. In this system, embryos were isolated from the mother before budding and were grown in a culture bottle until the formation of a rudimentary kidney so that it could be further developed by organ culture in vitro [43]. Using this combination, rudimentary kidneys continued to grow in vitro, as assessed by the observation of fine tubulogenesis and ureteric bud branching, indicating that the metanephros can finish developing ex utero even if the embryo is dissected prior to sprouting of the ureteric bud.

Based on these results, hMSCs were microinjected at the site of budding and subjected to relay culture. Before injection, the hMSCs were genetically engineered to express GDNF temporally using adenovirus and were labeled with the LacZ gene and dioctadecyl-3,3,3',3'tetramethylindocarbocyanine. Viral free manipulation can also be performed using thermoreversible GDNF polymer [44]. Soon after injection, the embryos, together with the placenta, were transferred to the incubator for whole embryos. After the relay culture, X-gal-positive cells were scattered throughout the rudimentary metanephros and were morphologically identical to tubular epithelial cells, interstitial cells, and glomerular epithelial cells [43]. In addition, reverse transcription-polymerase chain reaction revealed the expression of several podocyte- and tubulespecific genes [43]. These data demonstrated that using a xenobiotic developmental process for growing embryos allows endogenous hMSCs to undergo an epithelial conversion and be transformed into an orchestrated nephron consisting of glomerular epithelial cells (podocytes) and tubular epithelial cells that are linked. hMSCs can also differentiate into renal stroma after renal development [43].

We then examined the next issue in the successful development of an artificial kidney de novo: urine production. This requires that the kidney formed have the vascular 


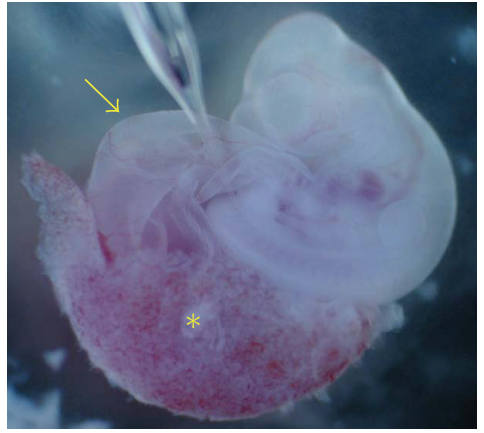

(a)

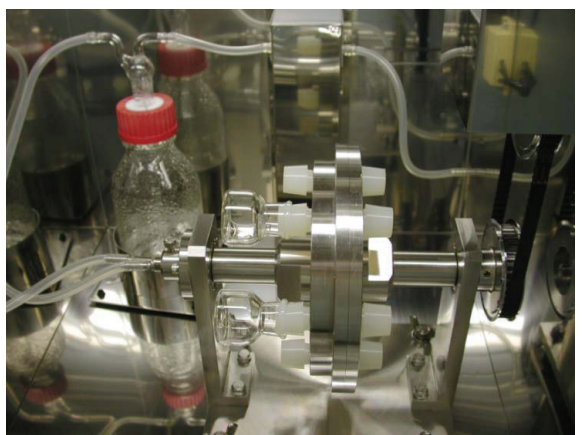

(b)

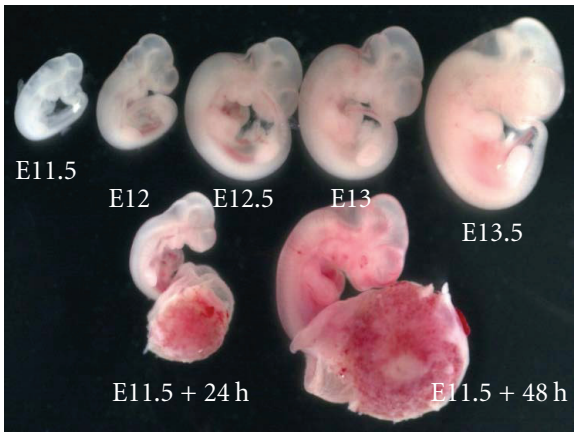

(c)

FIGURE 1: Strategy for nephron regeneration. MSCs were injected at the site of sprouting of the ureteric bud of E11.5 rat embryo (a). These embryos were developed in the whole-embryo culture system (b) for 48 hours. Embryos developed in this system shows the continuation of development in this system ((c): arrow, yolk sac; asterisk, placenta).
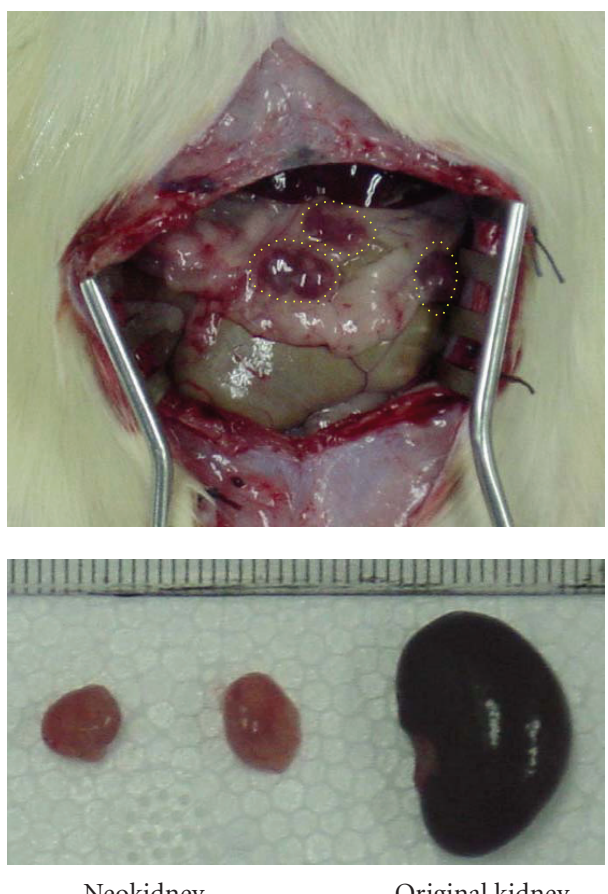
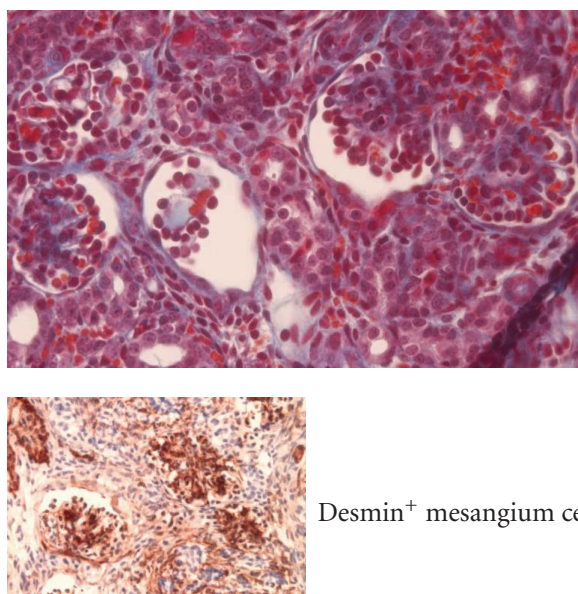

Desmin $^{+}$mesangium cells
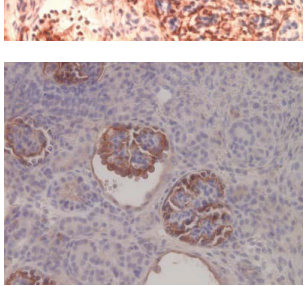

Synaptopodin ${ }^{+}$podocytes

FIgURE 2: Development of neokidney derived from hMSCs. Kidney anlagen derived from MSCs was transplanted into rat omentum. After this relay culturing, hMSCs formed into neokidney in the rat omentum. Histological analysis revealed a well-differentiated kidney structure (right panels). Desmin-positive mesangial cells and synaptopodin-positive podocytes can be seen (right panels).

system of the recipient; therefore, the primary system must be modified to allow for vascular integration from the recipient to form a functional nephron. We used the findings of Rogers et al. [8] described above, according to which the metanephros can grow and differentiate into a functional renal unit with integration of recipient vessels if it is implanted into the omentum. We confirmed that the omentum is a suitable site for vascular integration by comparing several transplantation sites [45]. We transplanted metanephroi from different embryonic stages into the omentum and found after 2 weeks that only metanephroi from rat embryos older than embryonic day 13.5 developed successfully. Therefore, the relay culture system was modified such that organ culture was terminated within 24 hours, by which time the metanephros was allowed to develop sufficiently and the kidney primordia could be transplanted into the omentum (termed "modified relay culture system"). As a result, an hMSC-derived "neo-" kidney was generated that was equivalent to a human nephron (Figure 2) [46].

To examine the origin of the vasculature in the neokidney, we generated LacZ-transgenic rats [47] as recipients so that donor- and recipient-derived tissues would be distinguishable by X-gal assay. The usefulness of genetically marked transgenic (tg) rats in organogenesis has been confirmed previously, using GFP as a marker, in the rat. Thus, we chose to use the $L a c Z$ tg rat, which expresses the marker gene ubiquitously, to determine the vascular origin in our experiments [47]. Using the modified relay 


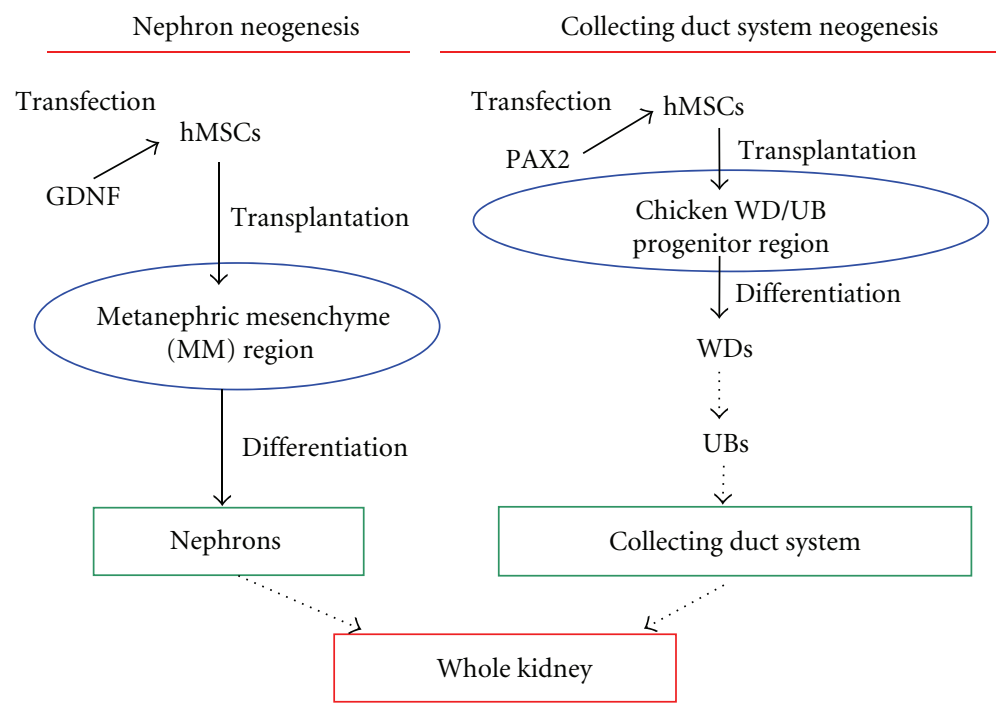

FIgURE 3: Dual step for the development of whole functional kidney.

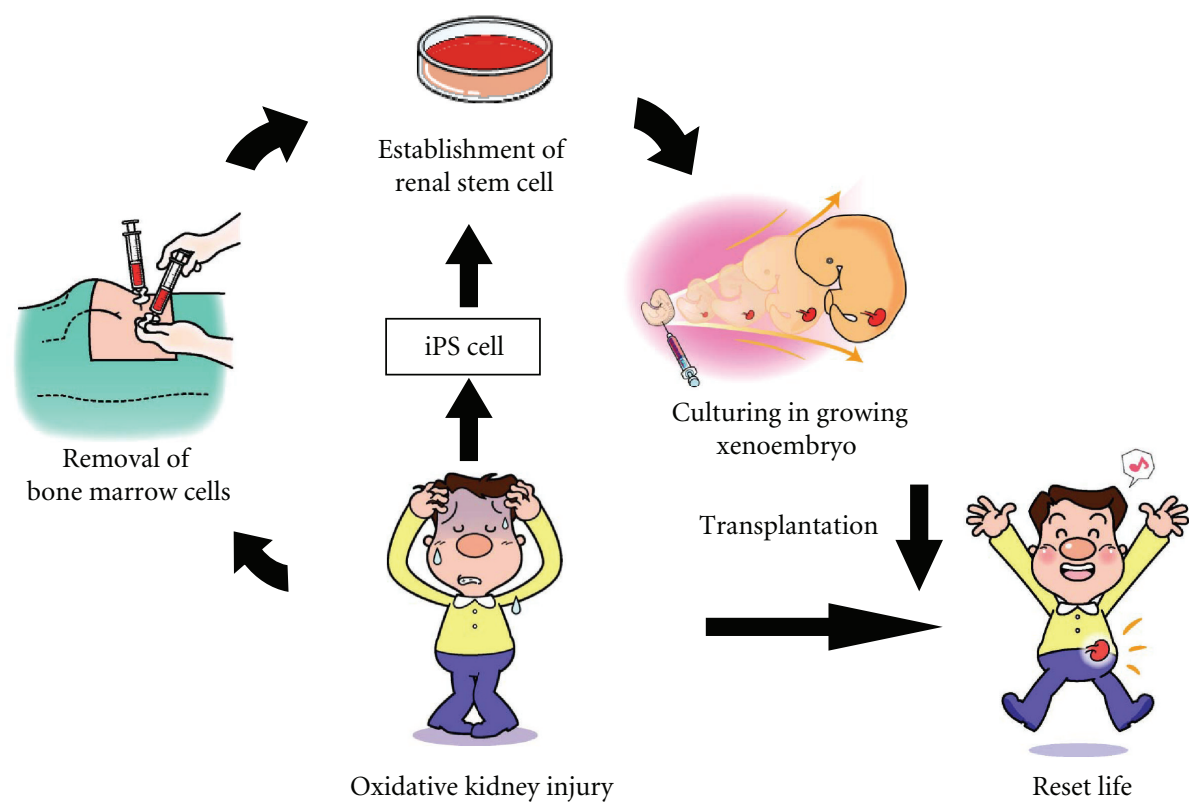

Figure 4: Putative scenario for the application of stem cell biology to kidney regeneration. Renal stem cells derived from bone marrow cells or skin fibroblasts of a dialysis patient are cultured in a growing xenoembryo for a given time to develop into kidney primordia, followed by their autologous implantation into the omentum of the same patient. Kidney primordia eventually become a self-organ that performs mature renal functions. The patient may experience relief from dialysis and completely overcome the renal disease.

culture, we found that several vessels from the omentum appeared to be integrated into the neokidney, and X-gal staining showed that most of the peritubular capillaries were LacZ positive, suggesting that they were of recipient origin. Furthermore, electron microscope analysis revealed red blood cells in the glomerular vasculature. These data indicated that the vasculature of the neokidney in the omentum originated from the host and communicated with the host circulation, suggesting its viability for collecting and filtering the host blood to produce urine. To verify this, we left the neokidney in the omentum for 4 weeks to develop further. What is surprising is that the structure developed hydronephrosis, confirming the ability of the neokidney to produce urine. If the ureters were buried under the fat of the omentum, the urine would have no egress, resulting in hydronephrosis. Analysis of the liquid from the expanded ureter showed higher concentrations of urea nitrogen and creatinine than the recipient sera in the native urine. Therefore, we concluded that the neokidney that developed in the omentum was capable of producing urine by filtering the recipient's blood.

Production of erythropoietin (EPO) to maintain erythropoiesis is another important function of the kidney. EPO stimulates red blood cell production and is produced 
mainly in the kidneys. We found 3 major findings regarding the hMSC-derived neokidney in rats: (i) human EPO is produced in rats harboring an organoid derived from autologous human bone marrow cells, (ii) human EPO production is stimulated by the induction of anemia, suggesting that this system preserves the normal physiological regulation of EPO levels, and (iii) levels of EPO generated by the neokidney in response to anemia in rats in which native EPO was suppressed are sufficient to restore red cell recovery to a rate similar to that in control rats [48]. Taken together, these data suggest that the neokidney derived from hMSCs may be able to fulfill all renal functions, including urine production. This work could lead to a new generation of therapy modalities for kidney disease.

As mentioned earlier, kidney is formed by a reciprocal interaction between the metanephric mesenchyme and the ureteric buds and is, thus, derived from a collecting system (such as the ureters and the collecting ducts), which is a derivative of the ureteric bud, and other components (such as the glomeruli and tubules, which are derivatives of the metanephric mesenchyme). Our current system may regenerate the derivatives of the metanephric mesenchyme but not the derivatives of the ureteric bud. Concerning this issue, we assessed whether MSCs are able to differentiate into the ureteric bud progenitor; we did this using chick embryos, which can be more easily manipulated and cultured as compared to mammalian embryos [49]. When hMSCs expressing Pax 2 were transplanted into the chicken ureteric bud progenitor region, they migrated caudally with the elongating Wolffian duct and were integrated into the Wolffian duct epithelia and then expressed LIM1, showing that they can differentiate into the Wolffian duct cells under the influence of local xenosignals [49]. This suggests that the whole kidney may be rebuilt by transplanting hMSCs at the suitable time and part for derivatives of the metanephric mesenchyme and ureteric bud (Figure 3).

Based on our successes, we are currently investigating the possibility of experimenting on a larger animal, that is, the pig, because the porcine kidney has almost the same volume as the human kidney. The ultimate size of the developed metanephros appears to be imprinted during the very early stages of development in the host embryo, as the metanephroi of larger animals transplanted into the omenta of smaller hosts develop into organs of larger volume (diameter and weight) as compared to that of a normal host kidney [50]. We hope that this system will facilitate the development of larger organs that are more suitable for use in humans (Figure 4).

\section{Conclusion}

In this article, we reviewed recent research on using renal stem cells to treat kidney diseases and proposed their possible application in kidney regeneration. We know that prior to the total loss of renal structure, kidney function can be restored by reactivating quiescent renal stem cells and/or supplying renal stem cells expanded sufficiently in vitro. Such stem cells may contribute to kidney regeneration, leading to recovery from organ failure.

We believe that emerging knowledge of kidney stem cell biology and developmental biology will enable the development of new therapeutic strategies for kidney regeneration that aim to regain damaged components of the kidney or restore kidney function.

\section{References}

[1] S. Kale, A. Karihaloo, P. R. Clark, M. Kashgarian, D. S. Krause, and L. G. Cantley, "Bone marrow stem cells contribute to repair of the ischemically injured renal tubule," Journal of Clinical Investigation, vol. 112, no. 1, pp. 42-49, 2003.

[2] F. Lin, K. Cordes, L. Li et al., "Hematopoietic stem cells contribute to the regeneration of renal tubules after renal ischemia-reperfusion injury in mice," Journal of the American Society of Nephrology, vol. 14, no. 5, pp. 1188-1199, 2003.

[3] F. Lin, A. Moran, and P. Igarashi, "Intrarenal cells, not bone marrow-derived cells, are the major source for regeneration in postischemic kidney," Journal of Clinical Investigation, vol. 115, no. 7, pp. 1756-1764, 2005.

[4] J. S. Duffield, K. M. Park, L. L. Hsiao et al., "Restoration of tubular epithelial cells during repair of the postischemic kidney occurs independently of bone marrow-derived stem cells," Journal of Clinical Investigation, vol. 115, no. 7, pp. 1743-1755, 2005.

[5] P. Semedo, M. Correa-Costa, M. A. Cenedeze et al., "Mesenchymal stem cells attenuate renal fibrosis through immune modulation and remodeling properties in a rat remnant kidney model," Stem Cells, vol. 27, no. 12, pp. 3063-3073, 2009.

[6] T. C. Chan, T. Ariizumi, and M. Asashima, "A model system for organ engineering: transplantation of in vitro induced embryonic kidney," Naturwissenschaften, vol. 86, no. 5, pp. 224-227, 1999.

[7] K. Okabayashi and M. Asashima, "Tissue generation from amphibian animal caps," Current Opinion in Genetics and Development, vol. 13, no. 5, pp. 502-507, 2003.

[8] A. S. Woolf, S. J. Palmer, M. L. Snow, and L. G. Fine, "Creation of a functioning chimeric mammalian kidney," Kidney International, vol. 38, no. 5, pp. 991-997, 1990.

[9] S. A. Rogers, J. A. Lowell, N. A. Hammerman, and M. R. Hammerman, "Transplantation of developing metanephroi into adult rats," Kidney International, vol. 54, no. 1, pp. 27-37, 1998.

[10] B. Dekel, H. Marcus, B. H. Herzel, W. O. Böcher, J. H. Passwell, and Y. Reisner, "In vivo modulation of the allogeneic immune response by human fetal kidneys: the role of cytokines, chemokines, and cytolytic effector molecules," Transplantation, vol. 69, no. 7, pp. 1470-1478, 2000.

[11] M. R. Hammerman, "Tissue engineering the kidney," Kidney International, vol. 63, no. 4, pp. 1195-1204, 2003.

[12] K. Osafune, M. Takasato, A. Kispert, M. Asashima, and R. Nishinakamura, "Identification of multipotent progenitors in the embryonic mouse kidney by a novel colony-forming assay," Development, vol. 133, no. 1, pp. 151-161, 2006.

[13] M. Unbekandt and J. A. Davies, "Dissociation of embryonic kidneys followed by reaggregation allows the formation of renal tissues," Kidney International, vol. 77, no. 5, pp. 407-416, 2010.

[14] H. C. Ott, T. S. Matthiesen, S. K. Goh et al., "Perfusiondecellularized matrix: using nature's platform to engineer 
a bioartificial heart," Nature Medicine, vol. 14, no. 2, pp. 213 221, 2008.

[15] B. E. Uygun, A. Soto-Gutierrez, H. Yagi et al., "Organ reengineering through development of a transplantable recellularized liver graft using decellularized liver matrix," Nature Medicine, vol. 16, no. 7, pp. 814-820, 2010.

[16] H. C. Ott, B. Clippinger, C. Conrad et al., "Regeneration and orthotopic transplantation of a bioartificial lung," Nature Medicine, vol. 16, no. 8, pp. 927-933, 2010.

[17] G. R. Martin, "Isolation of a pluripotent cell line from early mouse embryos cultured in medium conditioned by teratocarcinoma stem cells," Proceedings of the National Academy of Sciences of the United States of America, vol. 78, no. 12, pp. 7634-7638, 1981.

[18] L. M. Björklund, R. Sánchez-Pernaute, S. Chung et al., "Embryonic stem cells develop into functional dopaminergic neurons after transplantation in a Parkinson rat model," Proceedings of the National Academy of Sciences of the United States of America, vol. 99, no. 4, pp. 2344-2349, 2002.

[19] P. Blyszczuk, J. Czyz, G. Kania et al., "Expression of Pax4 in embryonic stem cells promotes differentiation of nestinpositive progenitor and insulin-producing cells," Proceedings of the National Academy of Sciences of the United States of America, vol. 100, no. 3, pp. 998-1003, 2003.

[20] M. Schuldiner, O. Yanuka, J. Itskovitz-Eldor, D. A. Melton, and N. Benvenisty, "Effects of eight growth factors on the differentiation of cells derived from human embryonic stem cells," Proceedings of the National Academy of Sciences of the United States of America, vol. 97, no. 21, pp. 11307-11312, 2000.

[21] T. Kobayashi, H. Tanaka, H. Kuwana et al., "Wnt4transformed mouse embryonic stem cells differentiate into renal tubular cells," Biochemical and Biophysical Research Communications, vol. 336, no. 2, pp. 585-595, 2005.

[22] B. M. Steenhard, K. S. Isom, P. Cazcarro et al., "Integration of embryonic stem cells in metanephric kidney organ culture," Journal of the American Society of Nephrology, vol. 16, no. 6, pp. 1623-1631, 2005.

[23] D. Kim and G. R. Dressler, "Nephrogenic factors promote differentiation of mouse embryonic stem cells into renal epithelia," Journal of the American Society of Nephrology, vol. 16, no. 12, pp. 3527-3534, 2005.

[24] C. Vigneau, K. Polgar, G. Striker et al., "Mouse embryonic stem cell-derived embryoid bodies generate progenitors that integrate long term into renal proximal tubules in vivo," Journal of the American Society of Nephrology, vol. 18, no. 6, pp. 1709-1720, 2007.

[25] E. A. Ross, M. J. Williams, T. Hamazaki et al., "Embryonic stem cells proliferate and differentiate when seeded into kidney scaffolds," Journal of the American Society of Nephrology, vol. 20, no. 11, pp. 2338-2347, 2009.

[26] R. P. Lanza, H. Y. Chung, J. J. Yoo et al., "Generation of histocompatible tissues using nuclear transplantation," Nature Biotechnology, vol. 20, no. 7, pp. 689-696, 2002.

[27] J. Chen, R. Lansford, V. Stewart, F. Young, and F. W. Alt, "RAG-2-deficient blastocyst complementation: an assay of gene function in lymphocyte development," Proceedings of the National Academy of Sciences of the United States of America, vol. 90, no. 10, pp. 4528-4532, 1993.

[28] M. S. Stern, "Chimaeras obtained by aggregation of mouse eggs with rat eggs," Nature, vol. 244, no. 5407, pp. 472-473, 1973.
[29] G. H. Zeilmaker, "Fusion of rat and mouse morulae and formation of chimaeric blastocysts," Nature, vol. 242, no. 5393, pp. 115-116, 1973.

[30] K. Takahashi and S. Yamanaka, "Induction of pluripotent stem cells from mouse embryonic and adult fibroblast cultures by defined factors," Cell, vol. 126, no. 4, pp. 663-676, 2006.

[31] K. Okita, T. Ichisaka, and S. Yamanaka, "Generation of germline-competent induced pluripotent stem cells," Nature, vol. 448, no. 7151, pp. 313-317, 2007.

[32] M. Wernig, A. Meissner, R. Foreman et al., "In vitro reprogramming of fibroblasts into a pluripotent ES-cell-like state," Nature, vol. 448, no. 7151, pp. 318-324, 2007.

[33] A. Meissner, M. Wernig, and R. Jaenisch, "Direct reprogramming of genetically unmodified fibroblasts into pluripotent stem cells," Nature Biotechnology, vol. 25, no. 10, pp. 11771181, 2007.

[34] J. Hanna, M. Wernig, S. Markoulaki et al., "Treatment of sickle cell anemia mouse model with iPS cells generated from autologous skin," Science, vol. 318, no. 5858, pp. 1920-1923, 2007.

[35] K. Takahashi, K. Tanabe, M. Ohnuki et al., "Induction of pluripotent stem cells from adult human fibroblasts by defined factors," Cell, vol. 131, no. 5, pp. 861-872, 2007.

[36] T. Kobayashi, T. Yamaguchi, S. Hamanaka et al., "Generation of rat pancreas in mouse by interspecific blastocyst injection of pluripotent stem cells," Cell, vol. 142, no. 5, pp. 787-799, 2010.

[37] S. Espejel, G. R. Roll, K. J. McLaughlin et al., "Induced pluripotent stem cell-derived hepatocytes have the functional and proliferative capabi lities needed for liver regeneration in mice," Journal of Clinical Investigation, vol. 120, no. 9, pp. 3120-3126, 2010.

[38] K. W. Liechty, T. C. Mackenzie, A. F. Shaaban et al., "Human mesenchymal stem cells engraft and demonstrate site-specific differentiation after in utero transplantation in sheep," Nature Medicine, vol. 6, no. 11, pp. 1282-1286, 2000.

[39] D. J. Prockop, "Marrow stromal cells as stem cells for nonhematopoietic tissues," Science, vol. 276, no. 5309, pp. 7174, 1997.

[40] L. Saxen, Organogenesis of the Kidney, Cambridge University Press, Cambridge, UK, 1987.

[41] J. A. Davies and C. E. Fisher, "Genes and proteins in renal development," Experimental Nephrology, vol. 10, no. 2, pp. 102-113, 2002.

[42] J. H. Lipschutz, "Molecular development of the kidney: a review of the results of gene disruption studies," American Journal of Kidney Diseases, vol. 31, no. 3, pp. 383-397, 1998.

[43] T. Yokoo, T. Ohashi, S. S. Jin et al., "Human mesenchymal stem cells in rodent whole-embryo culture are reprogrammed to contribute to kidney tissues," Proceedings of the National Academy of Sciences of the United States of America, vol. 102, no. 9, pp. 3296-3300, 2005.

[44] Y. Gheisari, T. Yokoo, K. Matsumoto et al., "A thermoreversible polymer mediates controlled release of glial cell line-derived neurotrophic factor to enhance kidney regeneration," Artificial Organs, vol. 34, no. 8, pp. 642-647, 2010.

[45] K. Matsumoto, T. Yokoo, S. Yokote et al., "Functional development of a transplanted embryonic kidney: effect of transplantation site," Journal of Nephrology. In press.

[46] T. Yokoo, A. Fukui, T. Ohashi et al., "Xenobiotic kidney organogenesis from human mesenchymal stem cells using a growing rodent embryo," Journal of the American Society of Nephrology, vol. 17, no. 4, pp. 1026-1034, 2006.

[47] H. Inoue, I. Ohsawa, T. Murakami et al., "Development of new inbred transgenic strains of rats with LacZ or GFP," 
Biochemical and Biophysical Research Communications, vol. 329, no. 1, pp. 288-295, 2005.

[48] T. Yokoo, A. Fukui, K. Matsumoto et al., "Generation of a transplantable erythropoietin-producer derived from human mesenchymal stem cells," Transplantation, vol. 85, no. 11, pp. 1654-1658, 2008.

[49] A. Fukui, T. Yokoo, K. Matsumoto, T. Kawamura, T. Hosoya, and M. Okabe, "Integration of human mesenchymal stem cells into the Wolffian duct in chicken embryos," Biochemical and Biophysical Research Communications, vol. 385, no. 3, pp. 330335, 2009.

[50] M. R. Hammerman, "Renal organogenesis from transplanted metanephric primordia," Journal of the American Society of Nephrology, vol. 15, no. 5, pp. 1126-1132, 2004. 


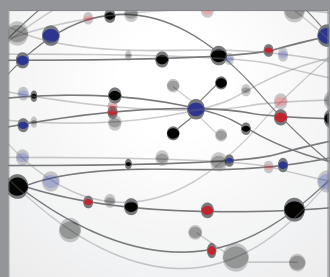

The Scientific World Journal
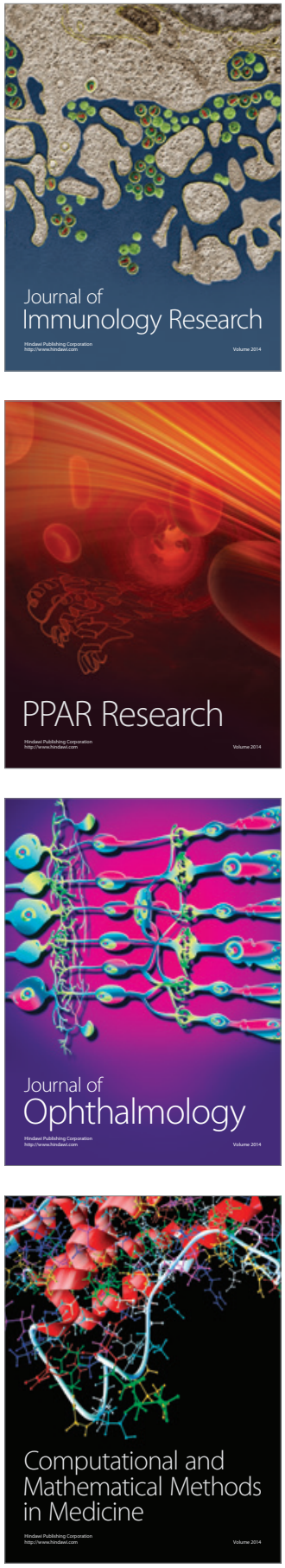

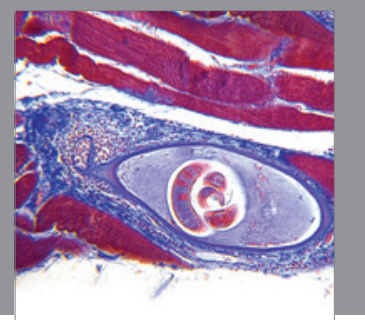

Gastroenterology

Research and Practice
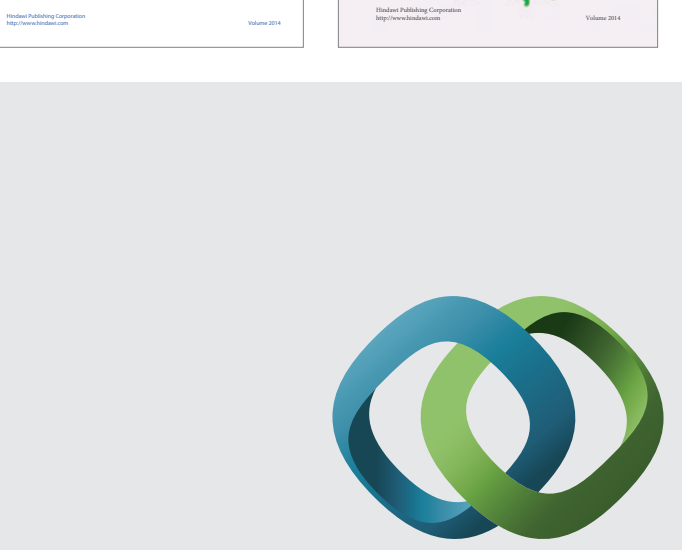

\section{Hindawi}

Submit your manuscripts at

http://www.hindawi.com
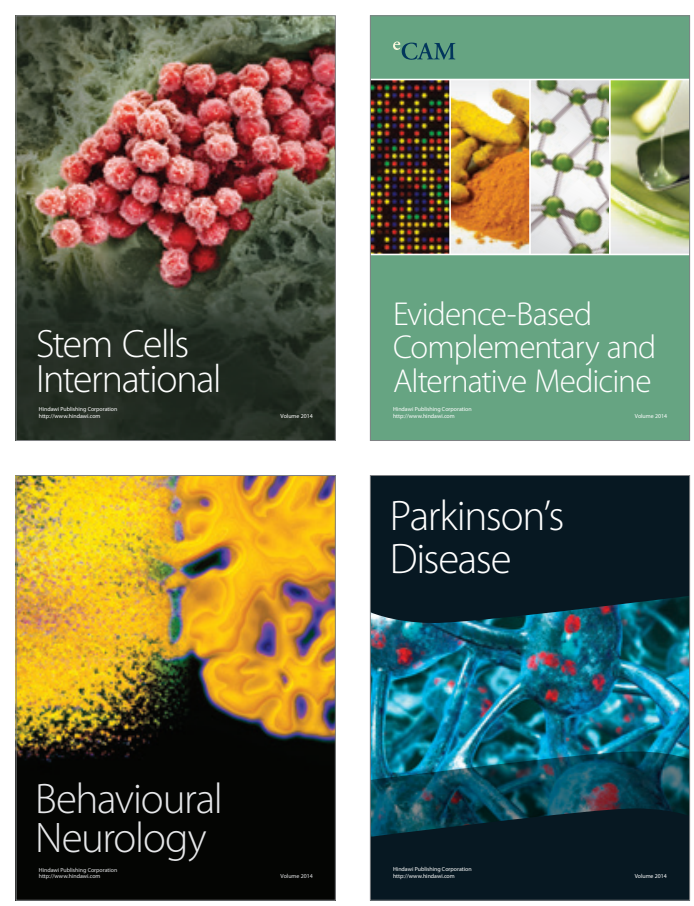

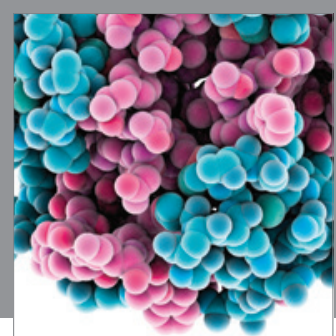

Journal of
Diabetes Research

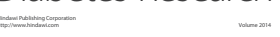

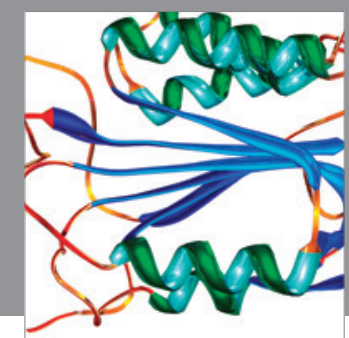

Disease Markers
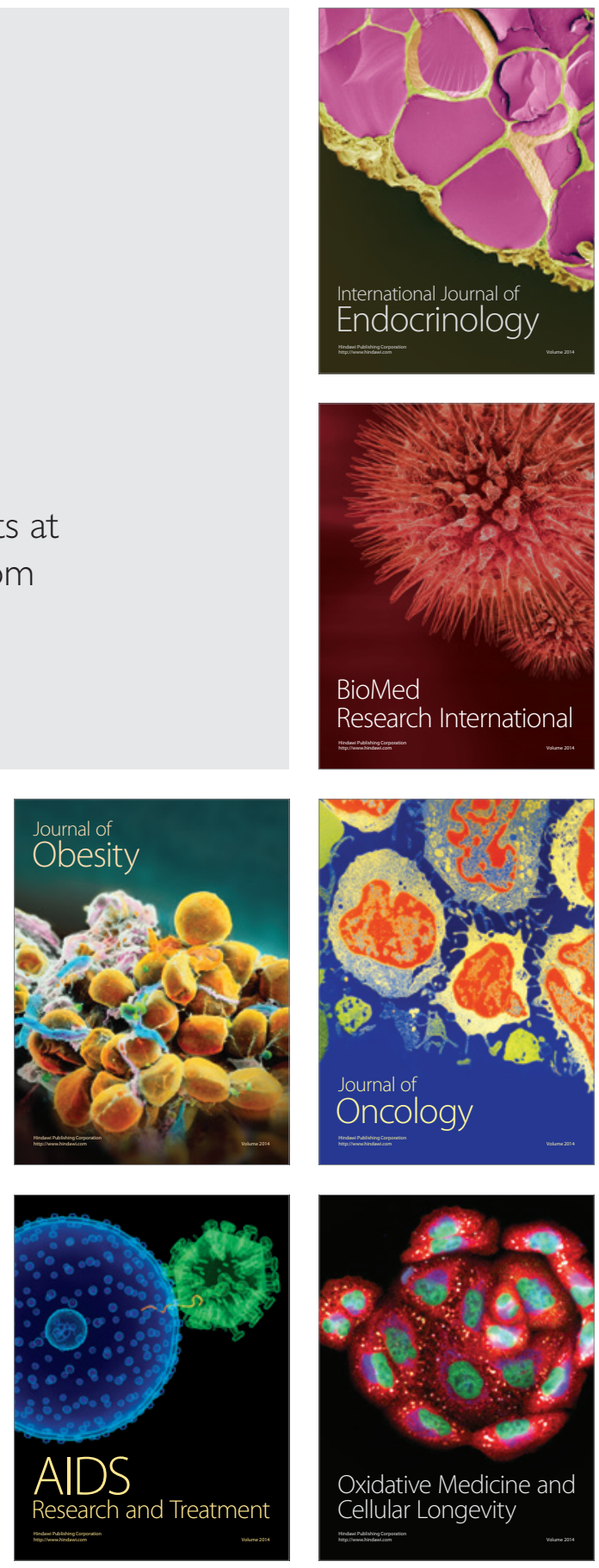